\title{
RECENT RESULTS ON ABUNDANCE DETERMINATIONS
}

\author{
SAUL J. ADELMAN* \\ Department of Physics, The Citadel, Charleston, SC 29409 USA
}

\begin{abstract}
I review abundance determinations of normal B5-F4 and peculiar stars published since 1984. Several analyses performed with photographic spectrograms indicate interesting stars which should be analyzed with high signal-to-noise data. Studies of stars of known ages which belong to clusters, associations, and moving groups should led to the most direct confrontations with theory. The increase in signal-to-noise ratio provided by electronic detectors with respect to photographic plates should allow accurate analyses of moderating rotating stars. High resolution, high signal-to-noise ratio studies have revealed crucial information about the line profiles of Sirius, Vega, and other A stars. It would aid comparison of analyses if we could agree on a standard set of gf-values and line damping constants. A computer bulletin board would be a useful means to provide and maintain such data as well as model atmosphere codes.
\end{abstract}

\section{INTRODUCTION}

I will discuss work published since 1984, when the last general CP star meeting was held at the Crimean Astrophysical Observatory. In the interim two important meetings were held related to my topic, the Workshop on Elemental Abundance Analyses (Adelman and Lanz 1988) and IAU Symposium No. 132, "The Impact of Very High S/N Spectroscopy on Stellar Physics" (Cayrel de Strobel and Spite 1988). Some of what I will say has been said in these proceedings. At this meeting there will be talks on related topics, so, for example, I will not deal with abundances from UV data nor in details of heavy element abundances.

I selected the literature from Astronomy and Astrophysics Abstracts section 114. Many papers are in a series of hopefully consistently performed analyses or study an element or elements in many stars. This is good as it is very difficult to judge the quality of single star analyses. I tend to ignore papers which used published data. The usefulness of analyses increases both with the number of consistently analyzed stars and with the well covered range of temperatures.

What is an A star? I could cover only those stars of spectral type A, both normal and peculiar. But some CP stars correspond to B stars and others to $F$ stars. Along the main sequence hotter than spectral type

*Guest Investigator, Dominion Astrophysical Observatory, Hertzberg Institute of Astrophysics, National Research Council of Canada 
B5, NLTE physics begins to become important for many atomic species. This defines a pragmatic upper temperature boundary near $16000 \mathrm{~K}$ although some CP stars are still hotter. Convective equilibrium rather than radiattve equilibrium dominates for stars cooler than the early $F$ stars. Thus I will consider stars only as cool as F4 or so although some Am stars may still be cooler. There are two subgroups with the hotter A stars having effective temperatures greater than $10000 \mathrm{~K}$. For these stars the convection zone high in the atmosphere does not extend to sufficient optical depth to cause a significant microturbulence in the line formation region.

\section{MODEST SIGNAL-TO-NOISE RATIO ANALYSES}

A decade ago most analyses were based on a few high dispersion photographic spectrograms each with signal-to-noise ratios of 20 at best. Since then we have the advent of computer graphics techniques to coadd and to measure spectra as well as a considerably greater usage of electronic detectors, especially Reticons and CCDs. I went the coadding spectrograms route. Later to work with variable stars I tried a Reticon at the Dominion Astrophysical Observatory. Was this conversion worth the effort? For electronic detectors one usually does not change the rather limited spectra region during the night while with plates one can get more than one complete spectrum of the same star. To obtain a complete spectrum of a star with coverage of some $950 \AA$ comparable to that with photographic plates takes 18 different settings and requires three or four years compared with two years at most with photographic plates. But, with the same exposure time and overhead, one gets a signalto-noise ratio of 200 rather than one with 80 at best.

I emphasize studies using spectra with signal-to-noise ratios of 50 or more. However, many studies are still based on a few plates. Still such studies can be useful especially as they can establish the general characteristics of the stars and indicate which ones should be studied further. Problems with our current analysis procedures once ameliorated will help to optimize the usefulness of the higher $\mathrm{S} / \mathrm{N}$ data.

\section{a. Normal Stars}

Ptitsyn and Ryabchikova (1986) performed a fine analysis of HR 8226 (B9.5 V) with three $9 \AA \mathrm{mm}^{-1}$ IlaO spectrograms as Cowley et al. (1982) suspected this star was iron-poor. But the abundances of nine elements including iron are close to solar. The derived microturbulence of $2 \mathrm{~km} \mathrm{~s}^{-1}$ is large compared to those I find for late normal B stars. Klochkova, Panchuk, and Tsymbal (1985) investigated the TH, Cr, and Fe abundances of the A2 V star $\theta$ Leo using data obtained at the Special Astrophysical Observatory. Savanov and Khalilov (1985) studied Vega and $\gamma$ Gem using several Crimean Astrophysical Observatory spectrograms. As also found by other investigators, $\gamma$ Gem is more metal rich than Vega. Savanov (1985) used model atmospheres techniques to study previously published data of $\eta$ Oph, $\varepsilon$ Oph, and $\theta$ Leo.

\section{b. Magnetic Ap Stars}

Analyses of the silicon stars HD 25823 ( $=41 \mathrm{Tau}$ ) and HD 26385 were performed by Bolcal, Koçer, and Duzgelan (1987) and by Bolcal et al. (1991), respectively, with $12.5 \AA \mathrm{mm}^{-1}$ spectrograms. The abundance 
anomalies of both stars are quite similar except for Eu II. Garcia and Levato (1986) studied the photometrically variable CP star HD 213918 using $16 \AA \mathrm{mm}^{-1}$ spectrograms. This helium deficient star is especially $\mathrm{O}, \mathrm{P}, \mathrm{Sc}, \mathrm{Tr}, \mathrm{Cr}, \mathrm{Mn}, \mathrm{Sr}, \mathrm{Zr}, \mathrm{Ce}$, and $\mathrm{Pr}$ overabundant. It may be a $\mathrm{Ti}-\mathrm{Sr}$ helium weak star. Savanov and Malanushenko (1991) reanalyzed the cool Ap star $\beta$ CrB using data by Adelman (1973) after attempting to correct for the companion. They also re-examined the U II $\lambda 3859.58$ line as presented by Cowley, Aikman, and Fisher (1977). Budaj, Zboril, and Zverko (1991) analyzed 68 Ori (v sin $\left.i=80 \mathrm{~km} \mathrm{~s}^{-1}\right)$, which Cowley et al. (1969) classified as a mild Si star, using 9 A $\mathrm{mm}^{-1}$ spectrograms and model atmospheres. They found it to be mildly silicon poor.

\section{c. Metallic Lined Stars}

Koçer et al. (1988) studied the Am star HD 73730 with $12.5 \AA \mathrm{mm}^{-1}$ spectrograms. Its deficient light, normal iron peak, slightly overabundant sprocess, and overabundant heavy elements showed it was a mild member of this class. HD 73730 belongs to the Praesepe open cluster and thus has a known age since it left the ZAMS. Its initial composition unaffected by the Am phenomenon can be deduced (at least in principle) from the analyses of normal cluster members.

While we can learn a great deal from field stars, more critical confrontations with the theories of chemically peculiar stars will come from stars with known ages. Most stellar observers do not get as much telescope time as they would like and often they cannot get time on large telescopes. Further, to get greater signal-to-noise ratios than are possible with photographic plates, we also cut down on the number of spectrograms obtainable in a given time. However, we must study stars with known ages in clusters, associations, and moving groups.

van't Veer-Menneret, Coupry, and Burkhart (1985) differentially analyzed the Am star HR 178, using $12 \AA \mathrm{mm}^{-1}$ spectrograms, with respect to $15 \mathrm{Vul}$. Its Geneva and Strömgren photometric indices indicate it is somewhat evolved. Their fine analysis showed this star had typical abundance anomalies of the main sequence Am stars, namely, Ca and Sc underabundant, $\mathrm{Tl}, \mathrm{V}, \mathrm{Cr}$, and $\mathrm{Fe}$ overabundant, and $\mathrm{Ni}, \mathrm{Sr}, \mathrm{Y}$, and $\mathrm{Zr}$ strongly overabundant. Coupry, van't Veer-Menneret, and Burkhart (1986) present line identifications and equivalent widths for this analysis. van't Veer-Menneret, Burkhart, and Coupry (1988) found this star's rare earth anomalies were generally a factor of 20 greater than solar. They also utilized a $2.4 \AA \mathrm{mm}^{-1} \mathrm{DAO}$ spectrogram and considered hyperfine structure for selected atomic species, an effect which should be more generally included in abundance analyses.

Lane and Lester (1987) performed fine analyses of six Am and three normal control stars. At Kitt Peak they obtained $2.8 \AA \mathrm{mm}^{-1}$ nitrogen baked IIIaJ spectrograms in the blue and $4.2 \mathrm{~A} \mathrm{~mm}^{-1}$ nitrogen baked 127-04 and IlaF spectrograms in the yellow. They tried to select relatively blend-free lines by synthetic spectral techniques using the atomic data of Kurucz and Peytremann (1975). This is a worthy successor to Smith's (1971) 'classic' study of Am star abundances as Lane and Lester did as careful a job as they could. However, Bolcal, Koçer, and Adelman (1992) using coadded Dominion Astrophysical Observatory $2.4 \AA \mathrm{mm}^{-1}$ spectrograms found major discrepancies. The BKA and LL equivalent widths for $15 \mathrm{Vul}$ and $32 \mathrm{Aqr}$ show differences, respectlvely, of over $10 \%$ for lines stronger than $100 \mathrm{~mA}$ and over $20 \%$ for all lines. Further the BKA microturbulences are about $1 \mathrm{~km} \mathrm{~s}^{-1}$ greater than the $\mathrm{LL}$ values. 
The question of effective temperatures and surface gravities of $F$ stars is somewhat controversial. In part as Balmer line profiles are temperature indicators, it is a question of the callbration of the spectrograms. Problems with photographic plates are well known, but both Reticons and CCDs can also have problems. Reticons are most likely to record linear spectra. Coadding spectrograms and using Baker densities as I did with DAO spectrograms yields equivalent widths within $10 \%$ of those obtained with Reticons. Comparisons of high signal-to-noise spectra produced with electronic detectors using different high dispersion spectrographs show differences in line depths of up to order $5 \%$. This reflects in part the lack of well measured instrumental profiles which can be used to estimate the light scattered along (and perpendicular tol the spectrum.

High resolution high signal-to-noise ratio spectra also reveal another problem for some stars, namely where is the continuum. Reducing the noise in a complex spectrum may show that the high points lack systematics. Establishing continuua for 15 Vul and 32 Aqr was difficult, but $\tau$ UMa in the photographic is a worse problem. Some of us have been fooled by noisy spectrograms to believe that a continuum exists. Such stars may only be analyzable by spectral synthesis techniques.

Okyudo and Sadakane (1990) studied $\pi$ Dra, classifled by Gray and Garrison (1987) as A2mA3IV, and 68 Tau, a member of the Hyades, a blue straggler, and a prototype hot Am star. Their four IlaO Okayama Astrophysical Observatory spectrograms per star usually had a dispersion of $4.1 \AA \mathrm{mm}^{-1}$. They used the $2.4 \AA \mathrm{mm}^{-1}$ coaddition of o Peg (see Adelman and Lanz 1988) to select lines. Usually their equivalent width scale was similar to those of Wright et al. (1964), Smith (1972), and Savanov (1983). Their fine analysis confirmed that $\pi$ Dra was an Am stars whose abundance anomalies were systematically more positive than those of $68 \mathrm{Tau}$. Both stars have anomaly patterns simtlar to o Peg (Adelman 1988b).

Dobrichev, Ryabchikova, and Raikova (1987) analyzed the sharplined early A star $\theta$ Vir using five IIaO spectrograms primarlly with a reciprocal dispersion of $4.2 \AA \mathrm{mm}^{-1}$. Although enhanced $\mathrm{Ti}, \mathrm{V}, \mathrm{Sr}, \mathrm{Y}, \mathrm{Zr}$, and $\mathrm{Ba}$ abundances along with normal $\mathrm{Ca}$ and $\mathrm{Sr}$ abundances suggest it is a hot Am star, $\theta$ Vir is a complicated multiple star (Hoffleit 1982). Savanov (1987) reanalyzed 15 Vul using data in the literature.

\section{d. HgMn stars}

Dobrichev et al. (1989) attempted to analyze the SB2 star HR 4072 with two $4.2 \AA \mathrm{mm}^{-1}$ spectrograms from the National Astrophysical Observatory of the Bulgarian Academy of Sciences. This paper inadequately treats the effects of the companion.

Zochling and Muthsam (1987) investigated the sharp-lined HgMn star k Cnc using $2.4 \AA \mathrm{mm}^{-1}$ DAO spectrograms and Muthsam's (1979) extension of Kurucz's (1970) ATLAS5 program. This was the latest of a series of papers in which Muthsam and his colleagues attempted to improve the analyses of CP stars by including more extensive metal line blanketing. I am quite interested to compare these exploratory studies with Kurucz's new ATLAS9 code and its related programs.

Lyubimkov and Polosukhina (1988) used seven $2.5 \AA \mathrm{mm}^{-1}$ Special Astrophysical Observatory spectrograms obtained on a single night to study $\alpha$ And. Its rotation and its duplicity cause problems. Due to ambiguities in the determination of the effective temperature, they used two different model atmospheres for their analyses. Zborll (1990, 
1991) analyzed $\pi^{1}$ Boo using equivalent widths from $9 \AA \mathrm{mm}^{-1}$ spectra.

\section{e. Mt. Wilson Observatory Studies of Adelman and Colleagues}

With colleagues I extended my analyses of sharp-lined stars which were based primarily on well-widened $4.3 \AA \mathrm{mm}^{-1}$ IIaO Nitrogen baked spectrograms taken with the coude spectrograph of the 2.5-m telescope at Mt. Wilson Observatory. The spectrograms were traced mainly with a PDS microdensitometer. The intensity tracings of different exposures were compared as the continuua and the line profiles were marked. After the equivalent widths were measured with a planimeter, the individual plate values for a given line were averaged. Although many of the stars have subsequently been re-analyzed, there are still several for which these analyses are the most recent study. There are tracings of spectrograms of additional stars which could be profitably analyzed, see e.g. Garcia and Adelman (1992).

Adelman (1984) studied $v$ Her, particularly whether metal line blanketing could explain slight anomalies in its optical energy distribution and reanalyzed the 38 Dra data of Adelman and Sargent (1972). Adelman and Fuhr (1985) examined how the then new critical compilation of Fe II gf values by Fuhr, Martin, and Wiese (1988) affected the derived stellar abundances through both changes in the individual gf values and the determination of the microturbulence. Adelman (1986) analyzed the mid B to early A stars $\theta$ Leo, $\tau$ Her, 14 Cyg, and 5 Aqr, Adelman (1987) HR 6559 (A7 IV) and $\eta$ Lep (FO IV), and Koçer et al. (1987) the Am star 32 Aqr. Many normal stars have one or two significant abundance anomalies.

\section{STARS WITH MODERATE ROTATION}

Most published analyses concern relatively sharp-lined stars. This reflects to a large extent a necessity of life for the high dispersion spectroscopist over a decade ago. However, coadding spectrograms and the use of electronic detectors alleviates this problem to some extent. We do not know with certainty whether the sharp-lined stars at a given spectral type have abundances typical of similar stars over the full range of observed rotational velocities. I note the interest of Ansari (1992) in this problem. With high signal-to-noise high dispersion spectrograms such questions can be studied. Thus a necessary program for the next decade is high quality abundance analyses of moderately rotating stars ( $v$ sin $i$ between 25 and $75 \mathrm{~km} \mathrm{~s}^{-1}$ ). Two studies by Lambert and his students indicate that such a program is possible. Although they worked with selected lines, synthetic spectrum techniques with improved gf-values should make a larger number of lines analyzable.

Venn and Lambert (1990) analyzed the $\lambda$ Boo stars, $\lambda$ Boo, 29 Cyg, and $\pi^{1}$ Orl with $v$ sin 1 values between 85 and $110 \mathrm{~km} \mathrm{~s}^{-1}$ and Vega using Reticon and CCD spectrograms covering approximately $13100 \mathrm{~A}$ intervals at a resolution of about $0.25 \mathrm{~A}$ at signal-to-noise ratios of 100 to 500 per resolution element. They selected lines with minimal blending or were blended by other lines of the same element. Model atmospheres analyses show that the metal deficiencies are more severe than previously reported with $[\mathrm{Fe} / \mathrm{H}]=-2.0$ to -1.3 . $\mathrm{Mg}, \mathrm{Ca}, \mathrm{T}$, and $\mathrm{Sr}$ are similarly underabundant with $\mathrm{Na}$ often having a smaller underabundances. $C, N, O$, and $S$ show near solar abundances. Vega is a mild $\lambda$ Boo star. The $\lambda$ Boo star abundance anomalies resemble those found for the 
interstellar gas.

Earlier Lambert, McKinley, and Roby (1986) studied the C I $9100 \AA$ lines in 22 early A type main sequence stars with $v$ sin 1 values between 15 and $177 \mathrm{~km} \mathrm{~s}^{-1}$. They used a Reticon, a resolution of 0.4 or $0.5 \AA$, and a typical signal-to-noise ratio of 150 . Their derived carbon abundances showed no significant trends with increasing rotation. This suggests that meridional mixing does not bring $\mathrm{CN}$ processed material into the atmospheres of these rotating stars.

\section{IV, SELECTED ELEMENTAL STUDIES}

\section{a. $\mathrm{HI}$}

Chalabaev et al. (1988) obtained Paschen $\beta$ and $\gamma$ and Brackett $\gamma$ line profiles of the AO IV star $\gamma$ Gem with the Fourier Transform Spectrometer at the CFH telescope. These lines are produced deeper in the atmosphere than the Balmer lines and probe the region of the temperature minimum. These IR lines show deep and narrow absorption core in good qualitative agreement with the predictions of NLTE model atmospheres. This work indicates NLTE effects may be important for A stars.

To check the accuracy of model atmospheres similar measurements need to be made for many more stars. Before invoking NLTE we must be certain that the hydrogen line broadening theory is adequate. Kamper and Beardsley (1987) found $\gamma$ Gem is a spectroscopic binary. The mass of $A$ is $3.6 \mathrm{M}_{0}$ and of $B$ is $1.0 \mathrm{M}_{0}$. The spectrum of $B$ has not been detected in the blue, but its lines are more likely to be seen in the red and infrared. If $B$ is a main sequence star, it is 4 magnitudes fainter than $A$.

b. $\mathrm{He} \mathrm{I}$

Wolff and Heasley (1985) measured the strengths of the He I $\lambda 4026$ and $\lambda 4387$ lines in main sequence $B$ stars in several associations and the field. They found problems with the line broadening theory of the later line. From $\lambda 4026$ alone $\mathrm{He} / \mathrm{H}=0.085$ with a $15 \%$ inaccuracy.

Dorichev et al. (1989) derived the He abundance of the HgMn star $\kappa$ Cnc using the atmosphere of Steptên and Muthsam (1987) and three $4.2 \AA$ $\mathrm{mm}^{-1}$ photographic spectrograms. They found $\mathrm{He} / \mathrm{H}=0.017$, three times greater than Adelman (1987b) who had superior data.

c. Li I

Burkhart et al. (1987) used the 1.4-m CAT and ESO coudé echelle spectrometer with a Reticon, a resolution of 100,000 , and a reciprocal dispersion of $1.9 \AA \mathrm{mm}^{-1}$ to observe $\lambda \lambda 6675-6725$ in a sample of Am, $\delta \mathrm{Del}$, and normal $F$ stars. The $\delta$ Del and Am stars show over-, normal, and underabundant LI abundances. Burkhart and Coupry (1991) reanalyzed these observations for consistency with those of Burkhart and Coupry (1989) who made similar observations of 5 sharp-lined Am Hyades stars and $\mu$ Ori with the CFH telescope and achieved a S/N of about 400 . The Hyades stars had cosmic LI abundances except for 16 OrI. They also derived abundances from $\mathrm{Al}, \mathrm{Si}, \mathrm{Ca}$, and $\mathrm{Fe}$ lines in the observed region.

Gerbaldi and Farragiana (1991) tried to clarify the nature of the observed features near Li I $\lambda 6708$ in the magnetic Ap stars $\beta$ CrB and $\alpha^{2}$ CVn. They compared CCD Cassegrain spectrograph spectra obtained with the 1.93-m telescope of the Observatoire de Haute Provence with 
synthetic spectra. For the former star LI may be up to 10 times overabundant, but the line is not quite at the laboratory wavelength while for the later star there are is no detection. A recent study of $L d I$ in early $F$ dwarfs is that of Balachandran (1990).

\section{d. Be II}

Griffin and Griffin (1986) obtained $0.83 \AA \mathrm{mm}^{-1} 3 \mathrm{~mm}$ wide spectrograms of Vega and Sirius with the Mount Wilson Observatory 2.5-m telescope's coude spectrograph. No features are at the position of Be II $\lambda 3131.06$ in both stars with formal upper limits on the equivalent widths of less than $1 \mathrm{~mA}$.

\section{e. $\mathrm{CNO}$}

Adelman, Hayes, and Philip (1986) used TI CCD \#3 with the KPNO coudé feed, camera 6 , and grating $\mathrm{C}$ to obtain observations in the red of the field Horizontal branch stars HD 109995 and HD 161817 and the normal A stars $\theta$ Leo and HR 6559. The FHB stars are both $O$ and $N$ poor while the normal stars are $\mathrm{N}$ normal and slightly $\mathrm{O}$ poor.

Roby and Lambert (1990) studied C, N, O abundances in 13 Am, 9 HgMn, 15 magnetic Ap, and 5 standard stars with coudé spectrometer of the McDonald Observatory and a Reticon. They usually obtained a wavelength coverage of $200 \AA$, a spectral resolution of $0.3 \AA$, and a signalto-noise ratio between 50 and 200 . Relative to the standard stars, they found following average abundances $[\mathrm{X} / \mathrm{H}]$ for $\mathrm{C}, \mathrm{N}$, and $\mathrm{O}$, respectively: Am stars $(-0.2,0.2,-0.4)$. HgMn stars (+0.1, -0.7, -0.3), SrCrEu stars $(-0.7$, $-0.6,-1.3)$, and Si stars $(-0.2,-0.2,-0.5)$. The $\mathrm{C}$ and $\mathrm{O}$ abundances roughly increase with surface temperature. The HgMn stars are markedly $\mathrm{N}$ deficient. I prefer different microturbulences and am concerned how the variability affects the results for the magnetic Ap stars. But these effects probably do not substantially change their results.

\section{f. N I and S I}

Sadakane and Okyudo (1989) observed 12 sharp-lined AO to A2 stars with a spectral resolution of $0.24 \AA$ and a CCD detector with the coudé spectrograph of the 1.9-m telescope of the Okayama Astronomical Observatory. They analyzed seven N I, a S I, and a Fe I lines near $8700 \AA$ using model atmospheres techniques and microturbulences from the literature which were not consistently determined. The normal stars exhibited nearly solar $\mathrm{N}$ and $\mathrm{S}$ while in the hot Am star o Peg $\mathrm{N}$ was slightly overabundant and in the two Ap stars $\mathrm{N}$ and $\mathrm{S}$ are both underabundant.

g. OI

van Santvoort (1987) obtained spectra of normal and peculiar B and A stars with the Reticon equipped Coude Echelle Spectrometer at the ESO 1.4-m CAT reflector. The resolving power was $\lambda / \Delta \lambda=10^{5}$. As is well known, the equivalent widths of the $O$ I triplet near $\lambda 7774$ in the peculiar stars are systematically smaller than the normal stars of corresponding spectral types. NLTE calculations were used to derive the abundances.

An extensive study of this O I triplet was also undertaken by Faraggiana et al. (1988) (normal stars), Gerbaldi et al. (1989) (Ap stars), and van't Veer-Menneret et al. (1989) (Am stars). A Reticon detector and 
the Cassegrain spectrograph of the 1.9-m telescope of the Observatoire de Haute Provence and a reciprocal dispersion of $50 \mathrm{~A} \mathrm{~mm}-1$ were used. As the triplet is blended, its total equivalent width was measured. NLTE and microturbulence effects could only be handled globally. The cool Ap stars show far greater underabundances of $O$ (up to a factor of 400) than do the HgMn stars. The Am stars typically show factors of 10 times underabundant.

\section{h. Ca II}

Guthrie (1987) dertved the abundance of calcium for a fairly complete sample of single-lined classical Am stars from the results of $\mathrm{K}$-line photometry and differential curve of growth analyses. A probable relationship between the age parameter $\delta_{\text {co }}$ and the calcium abundance is evidence for diffusion. I am concerned whether all Am stars have similar microturbulences and metallicities. K-line photometry samples just more than just the $K$ line. Berthet (1992) extended these results using $8 \AA \mathrm{mm}^{-1}$ OHP spectra which include Ca I $\lambda 4227$ as well as the $\mathrm{K}$-line. Observations of stars in three clusters confirms that the more an Am star is evolved the less it is calcium deficient.

\section{GaI}

Zverko and Zborll (1989) tried to derive the Gallium abundance of 53 Tau from Ga I $\lambda 4032.98$ and $\lambda 4172.06$. Fe II and TI II lines which blend with these lines, respectively, account for the observed equivalent widths.

\section{j. Rare Earths}

Magazzu and Cowley (1986) demonstrated that the abundances of the intermediate and light lanthanides in the Am star 32 Aqr are comparable in magnitude to those in the cool magnetic stars $10 \mathrm{Aql}$ and $\gamma \mathrm{Equ}$. DAO 2.4 A mm-1 spectrograms were used in this study. This result, which has important consequence for theory, confirms wavelength Coinc1dence Statistics studies. Later Cowley and Greenberg (1987) found that Er III was present in the magnetic star HR 465 and estimated an abundance two orders of magnitude less than results from Er II.

\section{k. Th II}

Lyubimkov and Savanov (1985) investigated four Am stars using 8 A $\mathrm{mm}^{-1}$ spectrograms. In $15 \mathrm{Vul}, 16 \mathrm{Or}$, and $63 \mathrm{Tau}$ they claim to have found the strong Th II line $\lambda 4019.13$. They first calculated synthetic spectra in an interval about this line for Procyon. Then to improve the fit they adjusted the gf values. This is a useful technique.

I compared the coadded spectrum of 15 Vul of Bolcal, Koçer, and Adelman (1992) with their plot. Our spectrum has at least twice the resolution and three times the signal-to-noise ratio used by Lyubimkov and Savanov. The feature they attribute to Th II $\lambda 4019.13$ appears to be centered at $\lambda 4019.067$. My colleagues and I identifled it as a blend of $F$ $I(219) 4019.05$ and $\mathrm{Ni} I(74) 4019.055$. There is only a suggestion of a slight longward asymmetry. To resolve this discrepancy requires a spectrim with at least our resolution and a signal-to-noise ratio of order 200 . 


\section{U II}

Iliev, Lyubimkov, and Savanov (1986) dertved the uranium abundance from the $\lambda 3859.58$ line using two $2.4 \AA \mathrm{mm}^{-1}$ and two $4.2 \AA \mathrm{mm}^{-1}$ spectrograms of the Ap star 73 Dra. They calculated synthetic spectra of Procyon to improve the gf values. Wahlgren, Leckrone, and Adelman (1989) disputed the finding which inspired this study.

\section{HIGH SIGNAL-TO-NOISE RATIO STUDIES}

\section{a. Line Profiles Studies}

Lines profiles have a major import for abundance analyses. For the magnetic Ap stars variable lines profiles can be used for Doppler imaging (see, eg, Hatzes 1991a, b, Rice and Wehlau 1991). Holweger, Gigas, and Steffen (1986) and Holweger, Steffen, and Gigas (1986) obtained spectra of 17 sharp-lined normal B9.5 V - A2 V stars with the ESO Coudé Echelle Spectrometer fed by the 1.4-m CAT with a resolution of 50,000 and a Reticon. They found significant star to star abundance variations, with those of the iron group and of magnesium or barium being correlated, with carbon varying independently. Many DAO Reticon spectra I have taken are of similar quality. Examining such spectra is an extraordinary learning experience.

Dravins, Lindegren, and Torkelsson (1990) analyzed a few photospheric Fe I and Fe II line proflles of Sirlus. They used the coudé echelle spectrometer at ESO in the double pass mode at a reciprocal dispersion of $0.1 \AA \mathrm{mm}^{-1}$. Their signal-to-noise ratio approached 1000 . They found $v$ $\sin 1 \approx 15.3 \pm 0.3 \mathrm{~km} \mathrm{~s}^{-1}$ with the profiles quite similar to Gaussians with full widths at half maximum of $\cong 8 \mathrm{~km} \mathrm{~s}^{-1}$ as well as to synthetic line profiles computed from hydrodynamical model atmospheres of Gigas. I am concerned slightly about their deconvolution procedure as deconvolution can have severe mathematical difficulties and introduce artifacts into deconvolved spectra when the signal-to-noise ratio is inadequate.

Further, the superposition of neighboring absorption lines produced spectral features which are much narrower than the widths of individual rotationally broadened profiles (Dravins et al. 1990). When I use the computer graphics spectral line measurement program of REDUCE (Hill and Fisher 1986) to break blends into components, such clues are vital to one's success. Increasing resolution and the signal-tonolse ratio aid this process. Often consideration experimentation is required as different trials can lead to variety of divisions. I was aware of these narrow features in the mid-1980's. Graham Hill was when I mentioned them to him. They show the importance of obtaining high dispersion spectra even for stars with some rotation.

Gulliver, Adelman, Cowley, and Fletcher (1991) obtained $2.4 \AA$ $\mathrm{mm}^{-1}$ Reticon spectra of Vega with the coudé spectrograph of the DAO 1.2$\mathrm{m}$ telescope and a mean signal-to-nolse ratio of $2500+$. Strong lines have classical rotational profiles with enhanced wings. Weak lines are clearly flat bottomed resulting in a trapezoidal appearance. Vega is not unique as $10 \mathrm{Tr}, v$ Cap, and $\beta$ PsA also show this behavior. These profiles may be signatures of rapidly rotating stars seen nearly pole on. Elste (1992) found evidence for latitude dependent effects in Vega consistent with this hypothesis. 


\section{b. Giant Stars}

Berthet (1990) used the coudé feed at Kitt Peak National Observatory to obtain CCD spectra of five regions at a reciprocal dispersion of $9.9 \mathrm{~A}$ $\mathrm{mm}^{-1}$ for five $\mathrm{F}$ giant stars whose blanketing parameters $\Delta \mathrm{m}_{2}$ are similar to those of Am stars. Spectroscopists had not considered HR 1676, HR 2880, HR 2927, HR 4042, and HR 4090 to be Am stars. But the analyses showed that they resemble $\delta$ Del stars (which have the Am star anomalies except for $\mathrm{Ca}$ and $\mathrm{Sc}$ which are normal) more than the Am stars. Their enhanced $\Delta \mathrm{m}_{2}$ can be explained in terms of $[\mathrm{Fe} / \mathrm{H}]$ as suggested by Hauck (1986). A second paper (Berthet 1991) based on ESO spectrograms showed that HR 6492, HR 8222, and HR 8392 with similar properties exhibited the same behavior.

These studies does not use what I consider to be the best gf values, which are usually those compiled by Wiese, Fuhr, and their associates at NIST. It would help the comparison of analyses if we could agree on a standard set of gf values. These could be updated from time to time so that one could say I used gf value set $\mathrm{xq}$. This problem is potentially far worse for synthetic spectral analyses. As a field we need a computer bulletin board to exchange data files, model atmospheres and related codes, and other important information.

\section{c. Field Horizontal Branch (FHB) A Type Stars}

Population II halo stars have considerably weaker lines than Population I stars of similar effective temperature and surface gravity. Their line weakness alone justifies obtaining at least moderately high signal-tonoise spectra. As the halo is an old population, most of its A type stars are on the horkontal branch. Those which have been studied at high dispersion are relatively nearby field stars. Adelman and Hill (1987) examined the FHB prototype A stars HD 109995 and HD 161817 as well as HD 64488, a slightly metal weak star with substantial rotation. The spectroscopic material for each star was at least $106.5 \AA \mathrm{mm}^{-1}$ IIaO DAO spectrograms coadded to increase the signal-to-noise ratio. That HD 109995 and HD 161817 have abundances similar to glants in globular clusters with blue horizontal branches and their spectrophotometric similarity to FHB stars in these clusters shows that they are bright analogs. HD 64488 has slightly less than solar abundances but is considerably more metal rich than the Population II stars. A number of such objects are now known. Their existence suggests that some star formation occurred in the Galactic Halo not that long ago.

Adelman and Phillp (1990a) used the KPNO coudé feed, CCD TI\#3, camera 5 , and grating $A$ with a resolution of $0.14 \AA$. Comparison of observations of HD 109995. HD 161817, and several normal stars with DAO spectra established the discrepancies between equivalent width scales. The FHB prototype HD 86986 has abundances almost identical with HD 109995 with a metallicity of about 1/200 solar. HD 130095 and HD 202759 are even more metal poor while HD 74721 and HD 161817 are slightly metal richer.

\section{d. Sirius}

Sadakane and Ueta (1989) analyzed the Kurucz-Furenlid (1978) atlas of Sirius which is based on $1.1 \AA \mathrm{mm}^{-1}$ spectrograms with a signal-to-noise ratio of $300+$. They found $\mathrm{Mg}, \mathrm{Al}, \mathrm{Si}, \mathrm{Mn}$, and $\mathrm{Ni}$ had solar abundances 
while $\mathrm{V}, \mathrm{Cr}, \mathrm{Sr}, \mathrm{Y}$, and $\mathrm{Zr}$ are definitely overabundant and $\mathrm{Ca}$ and Sc are underabundant, respectively, by 0.5 and $1.0 \mathrm{dex}$. Although fine analyses of such high quality material are useful, they should be regarded as preliminary to spectral synthesis analyses.

e. Vega

Gigas (1986) produced a very interesting study of the iron abundance of Vega using unpublished measurements by J. Mitton of Mt. Wilson Observatory spectrograms obtained by $R$. and $R$. Griffin. He found a substantial underabundance of $\mathrm{Fe},-0.55$ dex relative to the Sun. NLTE corrections for both Fe I and Fe II were calculated. Gigas (1988) extended his study by analyzing Mg I, Mg II, and Ba II lines. Mg is significantly underabundant while $\mathrm{Ba}$ is only mildly deficient with respect to the Sun. In many respects this is a remarkable study, but I am disturbed that the discrepancy between the abundances from Fe I and Fe II lines increases when NLTE corrections are applied. There may be problems or incompleteness in the atomic physics data or the stellar model. ATLAS9 models presumably can help to correct the later problem.

Adelman and Gulliver (1990) analyzed Vega using $2.4 \AA \mathrm{mm}^{-1}$

DAO Reticon spectra obtained by C. R. Cowley. The signal-to-noise ratio of this data was 1000 in this predecessor of the material obtained by Gulliver et al. (1991). Vega was found to be a metal-poor star with a mean underabundance of 0.60 . Later when the mean iron abundance from the fine analysis was used to generate a synthetic spectrum, we found the adopted value was a compromise to fitting the Fe I and Fe II lines as would be expected for a fast rotator seen pole on.

\section{f. Normal Stars}

Lemke $(1989,1990)$ analyzed the data of the two papers by Holweger et al. (1986). The Si, TI, and Fe abundances were correlated. Most stars had Fe abundances within $+/-0.30$ dex of solar, but Vega is an exception. The helium to hydrogen ratio is typically $1 / 10$. HR 5959 had unusual abundances (I noticed that this star may have flat bottomed lines in his illustration similar to those of Vega). The normal stars show a rather smooth transition of metallicity from those typical of Am stars to those which are relatively metal poor. This indicates a common physical origin for the abundances variability. especially for diffusion models with a range of weak stellar winds.

Adelman and Phillp (1992b) analyzed KPNO coudé feed, TI3 CCD, camera 5, and grating A data of a few spectral regions in the early A stars $\gamma$ Gem and 7 Sex and the late B star HR 5780. Their derived abundances are similar to those of other normal stars with similar temperatures. However, 7 Sex is an old disk horizontal branch star with a high proper motion and a radial velocity of $+97 \mathrm{~km} \mathrm{~s}^{-1}$.

\section{g. HgMn stars}

Adelman and Philip (1990b, 1992b) with simllar material studied the HgMn star HR 4817. Its elemental abundances are quite similar to the peculiar Mn star $53 \mathrm{Tau}$. It has a modest $\mathrm{Hg}$ II $\lambda 3984$ line which $53 \mathrm{Tau}$ lacks. Thus it may be a link between such stars and classical Mn stars. Takada-Hidal and Jugaku (1992) examined the blending of the Fe II $\lambda 6149$ line in the HgMn star $\mu$ Lep. The presence of a substantial Hg II 
$\lambda 6149$ line means that the ratio of Fe II $\lambda 6149$ to Fe II $\lambda 6147$ cannot be used to detect a magnetic field in this star.

\section{h. Adelman et al.'s DAO studies}

I am studying normal and CP stars with $2.4 \AA \mathrm{mm}^{-1} \mathrm{DAO}$ spectrograms. At present $12 \mathrm{HgMn}$ stars and 7 normal stars have been consistently analyzed (Adelman 1987b, 1988a, b, c, 1989, 1991, 1992a). Kocer et al. (1992) discuss a coolward extension which includes 2 normal $F$ and 2 Am stars. Analyses of Vega with Reticon data (Adelman and Gulltver 1990) and of $\alpha$ Dra as a test of coadding spectrograms (Adelman et al. 1987) are also consistent with these studies, but use a different wavelength region and have a lower $\mathrm{S} / \mathrm{N}$ ratio, respectively. Comparison of traditional line identification techniques with Wavelength Coincidence Statistics (Cowley and Adelman 1990) verified most of the results of these studies as well as demonstrated the complementanty of these methodologies. Cowley and Adelman (1992) examine possible correlations in these studies. Adelman and Hill 1987) discussed the coaddition of spectroscopic plates (see also Adelman and Lanz 1988) and analyzed three FHB stars in a consistent methodology.

The initial program consisted of the normal and HgMn stars that David S. Leckrone hoped to observe with the GHRS on the Hubble Space Telescope. It now contains more stars and involves both optical and ultraviolet data. The goal is to obtain consistent abundances for a wide stellar temperature range. Thus normal early B through late $F$ stars are being studied. It is very important to relate these values to those of the Sun. For pecullar stars I concentrated on single HgMn stars.

The study began with coadding spectrograms and later incorporated Reticon data. Fine analyses is the major analysis technique. Olga Pintado and I are working on early B stars, $\gamma$ Peg (B2 IV) and $\iota$ Her (B3 IV). As NLTE effects may be important, we are using theoretical studies to correct for them. Dursun Koçer. Çetin Bolcal, and I are studying normal F and Am stars (see Kocer et al. 1992). Their student Hulya Caliskan is studying a sharp-lined magnetic Ap star for her PhD thesis. Initially only sharp-lined stars were included. This requirement now has been relaxed. Further some double-lined HgMn binaries are being studied, for example 112 Her with Tanya Ryabchikova and her post graduate student Larisa Zakharova.

I am now beginning to use ATLAS9 model atmospheres. Glenn M. Wahlgren and I are re-evaluating the choice of model atmospheres, a study which also uses IUE low dispersion data recalibrated with the hot white dwarf calibration. This will lead to more consistent temperatures and surface gravities. It is unfortunate that there are no modern optical region spectrophotometric instruments with the precision and accuracy of the rotating grating scanners. What is needed is an automatic spectrophotometric telescope to produce flux curves of bright stars with photometric accuracy (Adelman 1992b).

Further we need improved gf values. That was a goal of my work on Vega. Austin Gulliver and I will use o Peg instead as the primary star for which we will try to derive astrophysical gf values. We intend to fit its spectrum as well as possible using spectrum synthesis techniques. Then we will use several other stars as checks. I do not see how without such methods one can obtain consistent abundances from early B through early G stars. 


\section{ACKNOWLEDGMENTS}

I thank those colleagues with whom I have interacted concerning problems related to abundance determinations in the last few years, especially Chris Aikman, Cetin Bolcal, Charles R. Cowley, Murray Fletcher, Wesley Fisher, Jeffrey R. Fuhr, Austin F. Gulliver, Graham Hill, Sveneric Johansson, Dursun Koçer, Robert L. Kurucz, Theirry Lanz, David S. Leckrone, William C. Martin, Arlene Musgrove, Scott W. Roby, Tanya Ryabchikova, and Glenn M. Wahlgren. I also want to thank those colleagues who sent me copies of their recent papers. I used the libraries of the Dominion Astrophysical Observatory and The Citadel in writing this paper. This work was supported in part by NASA Grant NAG 5-1551 and grants from The Citadel Development Foundation.

\section{REFERENCES}

Adelman, S. J. 1973, ApJ, 183, 95

Adelman, S. J. 1984, A\&AS, 58, 585

Adelman, S. J. 1986, A\&AS, 64, 173

Adelman, S. J. 1987a, A\&AS, 67, 353

Adelman, S. J. 1987b, MNRAS, 228, 573

Adelman, S. J. 1988a, MNRAS, 230, 671

Adelman, S. J. 1988b, MNRAS, 235, 749

Adelman, S. J. 1988c, MNRAS, 235, 763

Adelman, S. J. 1989, MNRAS, 239, 487

Adelman, S. J. 1991, MNRAS, 252, 116

Adelman, S. J. 1992a, MNRAS, in press

Adelman, S. J. 1992b, ASP Conf. Serles, 28, 159

Adelman, S. J., and Fuhr, J. R 1985, A\&A, 152, 414

Adelman. S. J., and Gulltver, A. F. 1990, ApJ, 348, 712

Adelman, S. J., and Hill, G. 1987, MNRAS, 226, 581

Adelman, S. J., and Lanz, T. eds. 1988, Elemental Abundance Analyses

(Lausanne, Institute of Astronomy, University of Lausanne)

Adelman, S. J., and Philip, A. G. D. 1990a, MNRAS, 247, 132

Adelman, S. J., and Philip, A. G. D. 1990b, A\&A, 238, 111

Adelman, S. J., and Philip, A. G. D. 1992a, MNRAS, 254, 539

Adelman, S. J., and Philip, A. G. D. 1992 b, PASP, 104, 316

Adelman, S. J., and Sargent, W. L. W. 1982, ApJ, 176, 671

Adelman, S. J., Bolcal, Ç., Koçer, D., and Inelman, E. 1987, PASP, 99, 130

Adelman, S. J., Hayes, D. S., and Philip, A. G. D. 1986, PASP, 98, 783

Ansari, S. G. 1992, A\&A, in press

Balachandran. S. 1990, ApJ, 354, 310

Berthet, S. 1990, A\&A, 227, 156

Berthet, S. 1991, A\&A, 251, 171

Berthet, S. 1992, A\&A, 253, 451

Bolcal, C., Koçer, D., and Adelman, S. J. 1992, MNRAS, in press

Bolcal, C., Koçer, D., and Duzgelen, A 1987, Ap\&SS, 139, 295

Bolcal, C., Koçer, D., Koktay, T., and Güzel, T. 1991, Ap\&SS, 185, 295

Budaj, J., Zborll, M., and Zverko, J. 1991, Bull. Astron. Inst.

Czechoslovakia, 42, 350

Burkhart, C., and Coupry, M. F. 1989, A\&A, 220, 197

Burkhart, C., and Coupry, M. F. 1991, A\&A, 249, 205

Burkhart, C., Coupry, M. F., Lunel, M., and van't Veer, C. 1987, A\&A, 172, 257

Cayrel de Strobel, G., and Spite, M. eds. 1988. The Impact of Very High S/N Spectroscopy on Stellar Physics (Dordrecht, Reidel) 
Chalabaev, A. A. Borsenberger, J., Malllard, J. P., and Praderie, F. 1988, in The Impact of Very High S/N Spectroscopy on Stellar Physics, eds. G. Cayrel de Strobel and M. Spite (Dordrecht, Retdel), p. 139

Coupry, M. F, van't-Veer-Menneret, C., and Burkhart, C. 1986, A\&AS, 64, 477

Cowley, A. P., Cowley, C., Jaschek, M., and Jaschek, C. 1969, AJ, 74, 375

Cowley, C. R., Aikman, G. C. L., and Fisher, W. A. 1977, Publ. Dom. Astrophys. Obs., 15, 37

Cowley, C. R., and Adelman, S. J. 1990, PASP, 102, 1077

Cowley, C. R., and Adelman, S. J. 1992, in preparation

Cowley, C. R, and Greenberg, M. 1987, PASP, 99, 1201

Cowley, C. R. Sears, R. L., Alkman, G. C. L, and Sadakane, K. 1982, ApJ. 254,191

Dobrichev, V. M., Klochkova, V. G., Panchuk, V. E., and Raikova, D. V. 1989, Bull. Spec. Astrophys. Obs. North Caucasus, 27, 3

Dobrichev, V. M., Raikova, D. V., Ryabchikova, T. A., and Topil'skaya, G. P. 1989, Astrofizika, 30, 91

Dobrichev, V. M., Ryabchikova, T. A., and Raikova, D. V. 1987. Astrofizika, 26, 55

Dravins, D., Lindegren, L., and Torkelsson, U. 1990, A\&A, 237, 137

Elste, G. H. 1992, ApJ, 384, 284

Faraggiana, R., Gerbaldi, M., van't Veer, C., and Floquet, M. 1988, A\&A, 201,259

Fuhr, J. R., Martin, G. A. and Wiese, W. L. 1988, J. Phys. Chem. Ref. Data 17. Suppl. 4

Garcia, Z. L., and Adelman, S. J. 1992, this meeting

Garcia, Z. L., and Levato, H. 1986, Astrophys. Lett., 25, 1

Gerbaldi, M., and Faraggiana, R. 1991, Mem. Soc. Astron. Ital., 62, 97

Gerbaldi, M., Floquet, M., Faraggiana, R., and van't Veer-Menneret, C. 1989, A\&AS, 81, 127

Gigas, D. 1986, A\&A, 165, 170

Gigas, D. 1988, A\&A, 192, 264

Gray, R. O., and Garrison, R. F. 1987, ApJS, 65, 581

Griffin, R, and Griffin, R. 1985, A8A, 149, 437

Gulliver, A. F., Adelman, S. J., Cowley, C. R., and Fletcher, M. F. 1991, Ap. J. 380,223

Guthrie, B. N. G. 1987, MNRAS, 226, 361

Hatzes, A. P. 1991a, MNRAS, 248, 487

Hatzes, A. P. 1991b, MNRAS, 253, 89

Hauck, B. 1986, A\&A, 155, 371

Hill, G., and Fisher, W. A. 1986, Publ. Dom. Astrophys. Obs., 16, 159

Hoffleit, D. 1982, The Bright Star Catalogue (Yale Untversity Observatory, New Haven)

Holweger, H., Gigas, D., and Steffen, M. 1986, A\&A, 155, 58

Holweger, H., Steffen, M., and Gigas, D. 1986, A\&A, 163, 333

Iliev, I. Kh., Lyubimkov, L. S., and Savanov, I. S. 1986, Astrofizika, 25. 237

Kamper, K., and Beardsley, W. R. 1987, AJ, 94, 1302

Klochkova, V. G., Panchuk, V. E., and Tsymbal, V. V. 1985, Bull. Spec. Astrophys. Obs. North Caucasus, 19, 22

Koçer, D., Adelman, S. J., Bolcal, C.., and Hill, 1992, this meeting.

Koçer, D., Bolcal, C., Inelmen, E., and Adelman, S. J. 1987, A\&AS, 70, 49

Koçer, D., Bolcal, C., Saglamsaatct, M., and Duzgelen, A. 1988, Ap\&SS, 149,273

Kurucz, R. L. 1970, Smithsonian Astrophys. Obs. Spec. Rep. 309

Kurucz, R. L., and Furenlid, I. 1979, Smithsonian Astrophys. Obs. Spec. 
Rep. 387

Kurucz, R L., and Peytremann, E. 1975, Smithsonian Astrophys. Obs. Spec. Rep. 362

Lambert, D. L., McKinley, L. K., and Roby. S. W. 1986, PASP, 98, 927

Lane, M. C., and Lester, J. B. 1987, ApJS . 65, 137

Lemke, M. 1989, A\&A, 225, 125

Lemke, M. 1990, A\&A, 249, 331

Lyubimkov, L. S., and Polosukhnta, N. S. 1988, Bull. Crimean Astrophys. Obs., 80, 30

Lyubimkov, L. S., and Savanov, I. S. 1975, Astrofizika, 22, 63

Magazzu, A. and Cowley, C. R. 1986, ApJ, 308, 254

Muthsam, H. 1979, A\&A, 73, 159

Okyudo, M., and Sadakane, K. 1990, PASJ, 42, 317

Ptitsyn, D. A., and Ryabchikova, T. A. 1986, AZh, 63, 527

Roby, S. W., and Lambert, D. L. 1990, ApJS, 73, 167

Rice, J. B., and Wehlau, W. H. 1991, A\&A, 246, 195

Sadakane, K., and Okyudo, M. 1989, PASP, 41, 1055

Sadakane, K., and Ueta, M. 1989, PASJ, 41, 279

Savanov, I. S. 1983, Bull. Crimean Astrophys. Obs., 66, 128

Savanov, I. S. 1985, Bull. Crimean Astrophys. Obs., 73, 92

Savanov, I. S. 1987, Bull. Crimean Astrophys. Obs., 76, 37

Savanov, I. S., and Khalilov, A. M. 1985, Bull. Crimean Astrophys. Obs., 72,106

Savanov, I. S., and Malanushenko, V. P. 1991, Astrofizika, 33, 251

Smith, M. A. 1971, A\&A, 11, 325

Smith, M. A. 1972 , A\&AS, 5,81

Steplén, K., and Muthsam, H. 1981. A\&A, 100, 159

Takada-Hidai, M., and Jugaku, J. 1992, PASP, 104, 106

van Santvoort. J. 1987, in Astrochemistry, eds. M. S. Vardya and S. P. Tarafdar (Dordrecht, Reidel), p. 391

van't Veer-Menneret, C., Burkhart, C., and Coupry, M. F. 1988, A\&A, 203, 123

van't Veer-Menneret, C., Coupry, M. F., and Burkhart, C. 1985, A\&A, 146, 139

van't Veer-Menneret, C., Faraggiana, R., Gerbaldi, M., Castelli, F., Burkhart, C., and Floquet, M. 1989, A\&A, 224, 171

Venn, K. A., and Lambert. D. L. 1990, ApJ, 363, 234

Wahlgren, G, M., Leckrone, D. S., and Adelman. S. J. 1989, BAAS, 21 , 1199

Wolff, S. C., and Heasley, J. N. 1985, ApJ, 292, 589

Wright, K. O., Lee, E. K., Jacobson, T. V., and Greenstein, J. L. 1964, Publ. Dom. Astrophys. Obs., 12, 173

Zochling, J., and Muthsam, H. 1987, A\&A, 176, 75

Zboril, M. 1990, Bull. Astron. Inst. Czechoslovakia, 41, 44

Zboril, M. 1992, Bull. Astron. Inst. Czechoslovakda, 42, 215

Zverko, J., and Zborll, M. 1989, Proceedings of the 8th meeting of the subcommittee "Magnetic Stars", Potsdam, p. 176 